\title{
Performance of the Thomson scattering diagnostic on Helical Symmetry Experiment
}

\author{
K. Zhai, ${ }^{\text {a) }}$ F. S. B. Anderson, K. Willis, K. Likin, and D. T. Anderson \\ HSX Plasma Laboratory, University of Wisconsin-Madison, Madison, Wisconsin 53706
}

(Presented on 20 April 2004; published 12 October 2004)

We report the design and the performance of the ten-point Thomson scattering system installed on Helical Symmetry Experiment (HSX). Based upon the design of the GA Thomson scattering system, the HSX Thomson scattering system covers the complete plasma cross section, providing a ten-point plasma parameter profile measurement during a single plasma shot. The system consists of a commercial $1 \mathrm{~J}-10 \mathrm{~ns}$ yttrium-aluminum-garnet laser, ten polychromators from GA, the specially designed collection optics, a CAMAC data acquisition unit, and a controlling computer. The high throughput collection optics enables measurement even at a plasma density of $0.5 \times 10^{12} \mathrm{~cm}^{-3}$. The system configuration, spectral response calibration, Raman calibration of the system, and operational results are presented. (C) 2004 American Institute of Physics. [DOI: 10.1063/1.1788835]

\section{INTRODUCTION}

The Thomson scattering experiment is a conventional diagnostic in fusion plasma research which provides measurement of the electron temperature and density. ${ }^{1-3}$ In the Helical Symmetry Experiment (HSX) plasma lab, a Nd: yttrium-aluminum-garnet (YAG) Thomson scattering system has been established and is now operation. The HSX Thomson scattering system uses the polychromator originally designed by General Atomics. ${ }^{1}$ Large collection optics are used in the system to get enough scattered photons from the HSX plasma with a typical density of $1 \times 10^{12} / \mathrm{cm}^{3}$. The system is capable of ten-point profile measurement. Ten identical fiber bundles with a transmission efficiency of 0.6 couple the collected photons to ten polychromators that disperse the collected light. The four wavelength channels in each of the ten polychromators are optimized for the temperature measurement range from $10 \mathrm{eV}-2 \mathrm{keV}$. A dedicated CAMAC system is used to record the data. The system has been spectrally and absolutely calibrated, which can provide routine profile measurement of the electron temperature and density.

\section{SYSTEM DESCRIPTION}

Physically, the HSX Thomson scattering system can be divided into several major elements. These are: laser system, beam transportation and stray light control, collection optics of the scattered light, spectral dispersion system, detection and signal handling, and control system. Each of these subsystems is interdependent, and the designs are dependent upon each other.

The HSX Thomson scattering system use a commercial manufactured Nd:YAG laser. This laser consists of an oscillator section and an amplification stage. The oscillator compartment is $Q$ switched by means of a Pockel cell and a Brewster polarizer. The beam from the oscillator passes

${ }^{a)}$ Electronic mail: kzhai@wisc.edu through a telescope and then enters into the amplifier compartment. The system being in service can deliver a pulse of energy $1 \mathrm{~J}$ with a $10 \mathrm{~ns}$ pulse width. The laser is located in a dust free air-conditioned room on an optical table. The pointing direction changes within 100 microdegrees for 3 months test.

The beam from the laser head is guided by three laser mirrors and then focused to the HSX vessel with a $3 \mathrm{~m}$ focal length lens. The spot size is around $1 \mathrm{~mm}$ in the plasma region of measurement, over vertically $20 \mathrm{~cm}$ extending from the geometrical center to the edge. In addition, a $1 / 2$ waveplate located in front of the laser head is used to adjust the beam polarization to enable maximum scattered light flux going into the collection optics. Entrance and exit tubes are specially designed with baffles to control the stray light. Entrance and exit windows are Brewster angle oriented fused silica windows to reduce the stray light and ensure maximum laser transmission. There is a silicon detector to monitor the reflected power from the entrance window. To accomplish the Brewster condition, we adjust the $1 / 2$ waveplate to minimize the reflected power from the entrance window. There are two charge coupled device cameras, one is located after the third mirror just before the beam entrance into the vessel and the other is located before the beam dump just after the beam leaving the vessel. These two cameras provide daily monitoring of the beam alignment with the collection optics. For safety reasons the beam is totally enclosed in a solid opaque material for this class four laser device.

Since the electron density in HSX is relatively low, typically around $1 \times 10^{12} / \mathrm{cm}^{3}$, it requires a special design of the collection optics to obtain enough signal level. We used ZEMAX® to design the collection optics, which allowed full optical design to accomplish the measurement requirements for the HSX experiment. The collection optics consists of two doublets made of BK7 and SF1 glass with antireblection coating for the wavelength from 800 to $1070 \mathrm{~nm}$. This collection lens collects scattering laser light from ten radial locations and images onto ten individual fiber bundles. A 


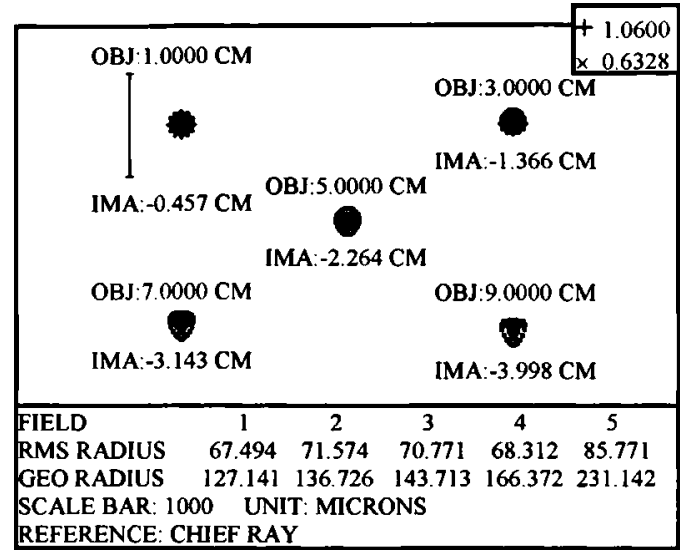

FIG. 1. Spot size on image plane, which shows the image size to be less than $100 \mu \mathrm{m}$ for all ten spatial channels, for wavelengths ranging from 650 to $1060 \mathrm{~nm}$.

$10 \mathrm{~cm}$ entrance diameter of the collection lens provides a collection of about 20000 photons at a density of 1 $\times 10^{12} / \mathrm{cm}^{3}$. The image space numerical aperture (NA) is 0.23 , enabling near perfect coupling to the fiber optics. The spot size on the image plane at the fiber bundle surface is less than $100 \mu \mathrm{m}$, as shown in Fig. 1. The collection lens is installed in a five-dimension adjustable holder, which is supported from the HSX lab floor without any physical contact with the vessel to ensure relative stability of the beam.

Ten identical fiber bundles are used to couple the collected scattered laser light to polychromator. Each bundle is $7 \mathrm{~m}$ long with a rectangular cross section at the collection optics image plane and a circular cross section at the polychromator input, and consists of 126 200/220-core/claddingsilica/silica low $\mathrm{OH}$ fibers with a NA between 0.24 and 0.25 . Figure 2 shows the rectangular end of the fiber bundle of $0.8 \mathrm{~mm} \times 7 \mathrm{~mm}$, which matches the image of the laser beam through the collection optics. We have tested the transmission efficiency of each individual bundle, showing that the transmission is equal to or greater than $60 \%$. Ten identical polychromators manufactured by GA are used to disperse the scattered laser light. There are four wavelength channels in each polychromator. The filters differentiating individual wavelength channels are optimized for the measurement of the electron temperature ranging from $10 \mathrm{eV}$ to $2 \mathrm{keV}$, appropriate for the measurement in both the edge and core

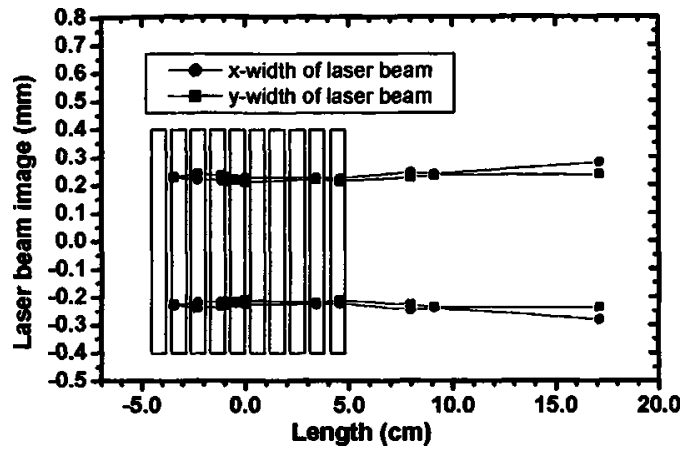

FIG. 2. Laser beam image on fiber surface. The ten rectangles of $0.8 \mathrm{~mm}$ $\times 7 \mathrm{~mm}$ indicate ten fiber surfaces on image plane. A margin of about $0.3 \mathrm{~mm}$ is for misalignment.

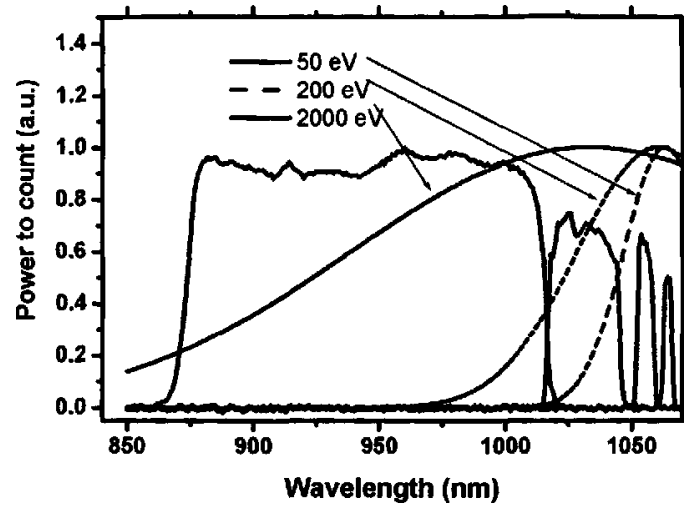

FIG. 3. Spectral response of the four channels of the polychromator. The $90^{\circ}$ Thomson scattering spectra at electron temperatures of 50, 200, and $2000 \mathrm{eV}$ are also shown.

region on HSX. The detectors and amplifiers are attached to the polychromators. The detector used is an EG\&G C30956E avalanche photodiode, which has a quantum efficiency greater than $40 \%$ around $1.06 \mu \mathrm{m}$ and an avalanche gain of 50-100. The output from the amplifier provides a signal in the range from 0.0 to $-1.0 \mathrm{~V}$. The signal is recorded by a gating Leroy Model 2250 charge integrating digitizer. The data acquisition system is a computer general purpose interface bus controlled CAMAC system dedicated to the HSX Thomson scattering experiment.

A HeNe laser is used for the alignment adjustment of the collection optics. This alignment laser can be aligned with the YAG laser in the laser room. An in-vessel target is designed to align the collection optics with the HeNe laser beam. The target is moved along the beam path and the scattered light is imaged on a three-fiber bundle. We maximize the signal collected by the center fiber to optimize the alignment of the collection optics with the laser beam.

There are two timing components in the system. A National Instrument 6602 timing card is used to set the time when the laser is fired during plasma discharge, and the time for taking background data. This provides an accuracy of 12.5 ns. Another timing component used is the Stanford DG535 unit, which provides the gate to the digitizer synchronized with the laser signal. This timing component provides a higher accuracy of about $1.5 \mathrm{~ns}$.

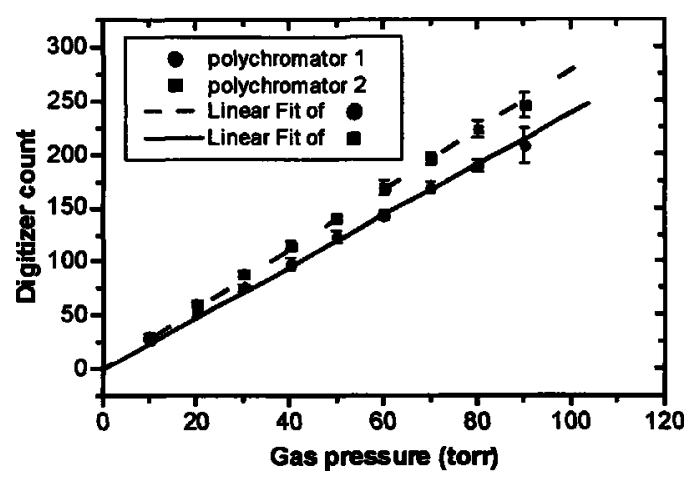

FIG. 4. Raman scattering signal changes with gas pressure. The calibration factor is defined by the slope of the fitted line. 


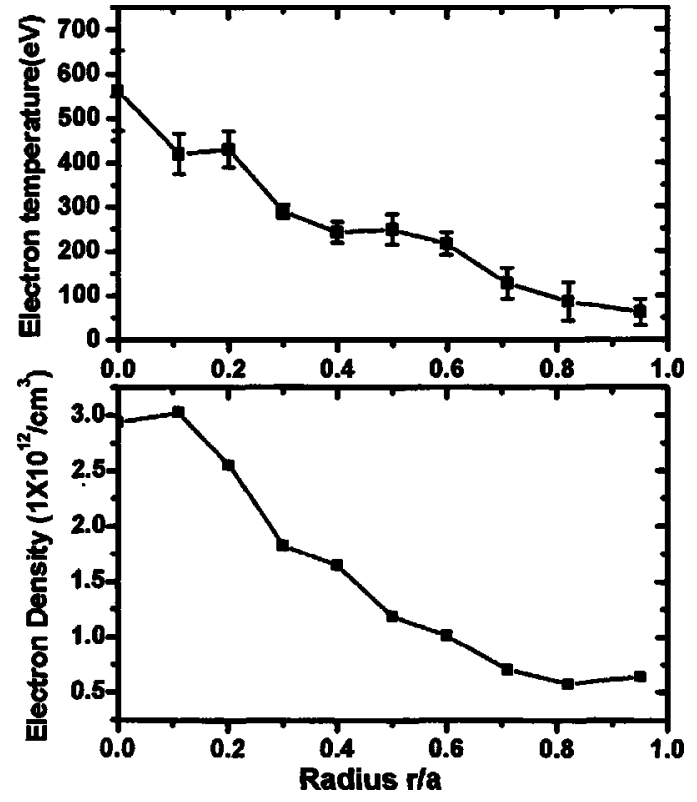

FIG. 5. Typical temperature and density profile measured for QHS central heating mode at $50 \mathrm{~kW}$ ECRH power.

\section{CALIBRATION}

To evaluate the measured signal quantitatively, the spectral sensitivity of the ten polychromators has to be calibrated in a calibration procedure with relatively high wavelength resolution. For this purpose we use a tungsten lamp as the light source which passes through a $1 / 4 \mathrm{~m}$ monochromator. The output from the exit slit is then split into two beams with a beam splitter, one of which is monitored with an absolutely calibrated InGaAs detector and the second beam is monitored with a silicon detector. Then we scan the wavelength from 850 to $1070 \mathrm{~nm}$ to calibrate the monitor silicon detector. After that we scan the wavelength again with the second beam from the monochromator exit fed into the polychromator. The result is shown in Fig. 3. We also include in the figure the scattered light spectrum at three electron temperatures of 50, 200, and $2000 \mathrm{eV}$.

Since the stray light contribution to the first wavelength channel makes the Rayleigh scattering troublesome, we use rotational Raman scattering for density calibration. ${ }^{4,5}$ For safety reasons, we choose nitrogen gas rather than hydrogen. We find the strongest lines from Raman scattering lie in the second wavelength channel extending from 1050 to $1060 \mathrm{~nm}$. We fill the HSX tank with nitrogen gas and change the gas pressure from 10 to 90 Torr. At each pressure we fire the laser and collect the scattered light the same way as we do the Thomson scattering experiment. The Raman scattering result is shown in Fig. 4.

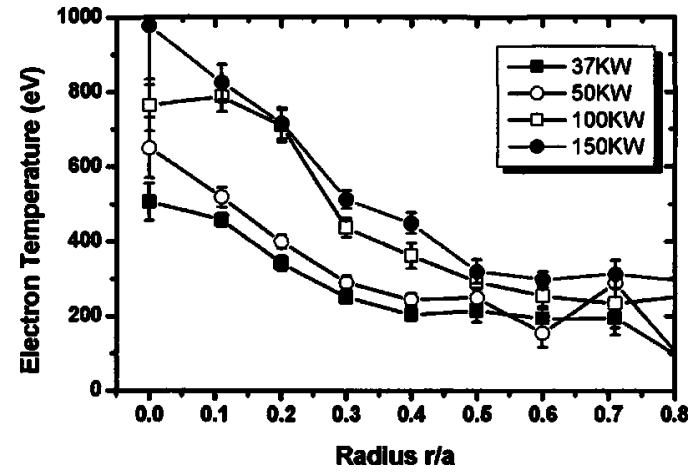

FIG. 6. Electron temperature profile changes with increasing of the ECRH heating power. These are QHS central heating shots at fixed density of 1.5 $\times 10^{12} \mathrm{~cm}^{-3}$.

\section{EXPERIMENTAL RESULTS AND FUTURE WORK}

Figure 5 shows a typical temperature and density profile measured by the HSX Thomson scattering for the QuasiHelical-Symmetry (QHS) mode at $50 \mathrm{~kW}$ electron cyclotron resonance heating (ECRH) power. We also carried out an ECRH power scan to study the plasma temperature response with different ECRH heating powers. Figure 6 shows the results of the power scan for the QHS mode. The lineaveraged electron density of the central channel measured by interferometer is fixed at about $1.5 \times 10^{12} \mathrm{~cm}^{-3}$. It is shown that the whole temperature profile increase with the heating power. The central temperature increases from about 500 to $950 \mathrm{eV}$ while the heating power increases from 37 to $150 \mathrm{~kW}$.

In summary, a Nd:YAG ten channel Thomson scattering system has been established and is now operational in the HSX plasma lab. It provides routine measurement of the electron temperature and density profile. Since the operational density in HSX is relatively low, the signal-to-noise ratio is of great importance for this particular case. Future work will concentrate on reducing the noise level and developing new data analysis techniques.

\section{ACKNOWLEDGMENT}

This work is supported by US DoE under Grant No. DE-FG02-93ER54222.

${ }^{1}$ T. N. Carlstrom, J. H. Foote, D. G. Nilson, and B. W. Rice, Rev. Sci. Instrum. 66, 493 (1995).

${ }^{2}$ C. J. Barth, F. J. Pijper, H. J. V. D. Meiden, J. Herranz, and I. Pastor, Rev. Sci. Instrum. 70, 763 (1999).

${ }^{3}$ K. Narihara, I. Yamada, H. Hayashi, and K. Yamauchi, Rev. Sci. Instrum. 72, $1122(2001)$

${ }^{4}$ J. Howard, B. W. James, and W. I. Smith, J. Phys. D 12, 1435 (1979).

${ }^{5}$ C. M. Penny, R. L. St. Peters, and M. Lapp, J. Opt. Soc. Am. 64, 712 (1974). 